Proceedings

\title{
Quality Control of Honey by Visible and Near Infrared Spec- troscopy (Vis-NIRS) and Headspace-Ion Mobility Spectrometry (HS-IMS)
}

\author{
José Luis P. Calle ${ }^{1, *}$, Marta Ferreiro-González ${ }^{1}$, María José Aliaño-González ${ }^{1}$, Estrella Espada-Bellido ${ }^{1}$, Miguel \\ Palma ${ }^{1}$ and Gerardo F. Barbero ${ }^{1}$
}

1 Department of Analytical Chemistry, Faculty of Sciences, IVAGRO, ceiA3, University of Cadiz, 11510 Puerto Real, Cadiz, Spain; joseluis.perezcalle@uca.es (J.L.P.C.); marta.ferreiro@uca.es (M.F.-G.); mariajose.alianogonzalez@alum.uca.es (M.J.A.-G.); estrella.espada@uca.es (E.E.-B.); miguel.palma@uca.es (M.P.); gerardo.fernandez@uca.es (G.F.B.)

* Correspondence: Jose Luis Pérez Calle (J.L.P.C.), Department of Analytical Chemistry, Faculty of Sciences, University of Cadiz, 11510 Puerto Real, Cadiz, Spain

Publisher's Note: MDPI stays neutral with regard to jurisdictional claims in published maps and institutional affiliations.

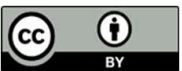

Copyright: (c) 2020 by the authors. Submitted for possible open access publication under the terms and conditions of the Creative Commons Attribution (CC BY) license (http://creativecommons.org/licenses/by/4.0/).

\begin{abstract}
Honey is a natural sweetener produced by bees from flower nectar. Based on the EU regulations, honey is a pure product so the addition of any other substance is prohibited. Nowadays, most of the analytical techniques used to detect honey frauds are expensive, time-consuming, destructive, and they require skilled operators. Hence, in the present work, visible and near infrared spectroscopy (Vis-NIRS) together with headspace-ion mobility spectrometry (HS-IMS) are proposed to determine the authenticity of honey. The obtained results demonstrate the potential of both techniques in combination with support vector machine (SVM) to detect honey adulterations.
\end{abstract}

Keywords: honey; adulteration; ion mobility spectrometry; visible and near infrared spectroscopy; chemometrics tools

\section{Introduction}

Honey is a product with a high nutritional value and beneficial properties due to its content in sugars, amino acids, organic acids, and biologically active compounds [1]. According to Codex Alimentarius the addition of any substance to honey is prohibited [2]. Moreover, its quality and composition depends on its botanical and geographical origins [3]. Thus, to ensure its quality, Protected Designations of Origin (PDO) and Protected Geographical Indications (PGI) have been established around the world [4].

Because of the high demand and price of honey in comparison to other sweeteners, it is one of the most likely food products to be targeted for adulteration. Among the most common adulterants are inverted sugar, rice syrup, maltose syrup or corn syrup, because of its low price and the similarity of its composition to honey [5]. This is an illegal activity and can also cause health problems in consumers since some of the ingredients used as adulterants may be allergens, becoming a potential risk for unaware consumers [6].

Different analytical techniques have been proposed for the detection of adulteration in honeys. These include high performance liquid chromatography (HPLC) [7], proton nuclear magnetic resonance (NMR) [8], gas chromatography with mass spectrometry [9] or based on DNA analysis [10]. Most of these methods are expensive, time-consuming, destructive, and require expert operators, which limit their use as routine analytical methods. For this reason, visible and near infrared spectroscopy (Vis-NIRS) [4,11] and headspace-ion mobility spectrometry (HS-IMS) [6,12] are currently proposed for the detection of adulterated honey. These techniques present several advantages as they allow the development of green, low cost and fast methods and both are easy to use. In addition, to 
automate the process, these techniques are combined with mostly parametric chemometric tools such as linear discriminant analysis (LDA). Non-parametric techniques such as support vector machines (SVM) have been used with very results for similar purposes $[13,14]$. Based on this, the aim of the present study is to determine the potential of HS-IMS and Vis-NIRS techniques in combination with SVM for the determination of adulterations in honey.

\section{Materials and Methods}

\subsection{Samples}

Multi-floral honey from the PDO of Granada (Lanjarón, Granada, Spain) was chosen as pure honey. As adulterants, five different types of common sweeteners were used: rice syrup (RS), brown cane sugar (BS), invert sugar (IS), fructose syrup (FS), and high fructose corn syrup (HFCS).

The adulterated samples were prepared in duplicate by adding to the pure honey each of the adulterants at ratios of: $5 \%, 10 \%, 20 \%, 25 \%, 30 \%, 40 \%$, and $50 \%$. So, a total of 70 adulterated samples was used in this study. Furthermore, two samples of pure honey and one of each pure adulterant were prepared in duplicate, so the final data matrix was made up of 84 samples.

\subsection{Analysis of the Sample}

All the samples were analyzed by HS-IMS and Vis-NIRS as previously described $[4,6]$.

\subsection{Data Analysis}

The Vis-NIR Spectra [4] and the Ion Mobility Sum Spectra (IMSS) [13] were obtained for all samples. For the present study, 3 different datasets arranged in matrices ( $\left.D_{n \times m}\right)$ were obtained, where $n$ was the number of honey samples and $m$ was the number of variables. The HS-IMS dataset is made up of 578 drift times (D84578), the Vis-NIRS dataset consists of 382 absorbance values (D84382), and the dataset from the joint of HS-IMS with Vis-NIRS has a total of 960 variables (578 drift times + 382 absorbance values (D84×960)). All chemometric analyses which include HCA (hierarchical cluster analysis) and SVM were performed by means of R-studio software (RStudio Team (2020), Boston, MA, USA).

\section{Results and Discussion}

The aim of this study was to demonstrate the effectiveness of HS-IMS, Vis-NIRS and, the combination of both techniques, to detect the adulteration of honeys. For this purposes, chemometric analyses were performed for each of the three datasets.

First, to determine if there is a tendency to group the samples according to the type of adulterant and the percentage used, an HCA (hierarchical cluster analysis) was carried out as an exploratory technique. This analysis was performed using the joint HS-IMS/VisNIRS dataset. TheWard's method and the Euclidean distance were used. This analysis is only performed on the joint dataset, because the grouping trend of the separate techniques has already been studied in previous studies $[4,13]$. In addition, the existence of a clustering trend in that dataset implies a tendency of group by the specific techniques. The results are shown in a circular dendrogram (Figure 1) to facilitate visualization due to the high number of samples. 


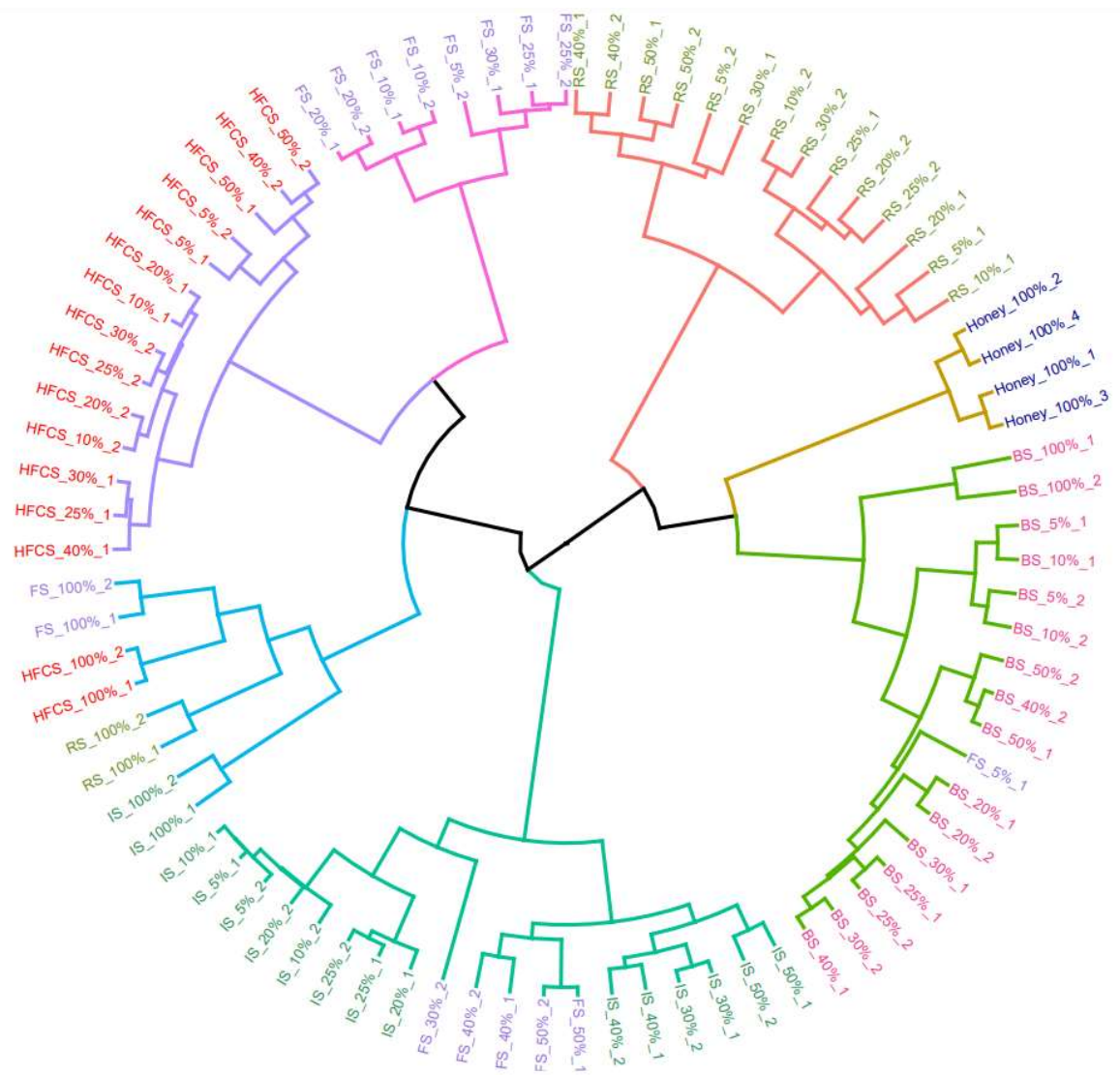

Figure 1. Dendrogram resulting from the HCA analysis using the joint HS-IMS/Vis-NIRS dataset. Ward's method and Euclidean distances were used. Samples are colored according to the type of adulterant in the sample: RS (light green), IS (dark green), BS (pink), FS (purple) and HFCS (red), and pure honey (dark blue).

As can be seen, there is a trend of the samples to be classified according to the type of adulterant and the percentage used. Focusing on the pure adulterants, all of them appear in the same cluster (blue coloured) except BS which is grouped in a different cluster (green coloured). However, in this green cluster appears all the samples adulterated with different percentage of BS, and the sample "FS_5\%_1". The replica of this sample is not contiguous, therefore it was assumed that there must be some mistake with this specific samples it was considered as an outlier. In the case of pure honey samples all of them appear in the same cluster (mustard coloured). Regarding the samples adulterated at different percentages, it is observed that HFCS samples appears in the same cluster (purple coloured), those adulterated with RS appear in the red cluster. Additionally, the samples containing BS appeared in the green cluster with the exception already mentioned. Nevertheless, samples adulterated with FS and IS seem to be more dispersed. Based on these results, it can be stated that there is a strong clustering trend depending on the adulterant used and to a lesser extent on the percentage of adulteration. Since there is a trend but not a perfect separation according to the type of adulterant, non-parametric supervised techniques such as the support vector machine (SVM) were then used.

SVM with radial basis function (RBF) was chosen as algorithm and six groups were considered a priori: one for the pure honey and one for each of the 5 adulterants used. Note that for this analysis and the rest of them, the sample "FS_5\%_1" was eliminated because it has been identified as an outlier. This algorithm has two hyperparameters to be optimized, gamma $(\gamma)$ that determines the behaviour of the kernel and, $\mathrm{C}$ that controls the 
penalty for misclassified samples. To optimize $C$ and $\gamma$, a grid search method with exponentially growing sequences of $C$ and $\gamma$ were applied. Therefore, values were taken every 0.5 units in the range $[-10,10]$ for $\log _{2} \mathrm{C}$ and $\log _{2} \gamma$. Each combination of parameter was checked using fivefold cross-validation and the one with the greatest accuracy was selected as optimal. This optimization process was carried out for the three datasets and Figure 2 shows the accuracy result for each combination. Furthermore, the best optimization values for each dataset are also displayed.
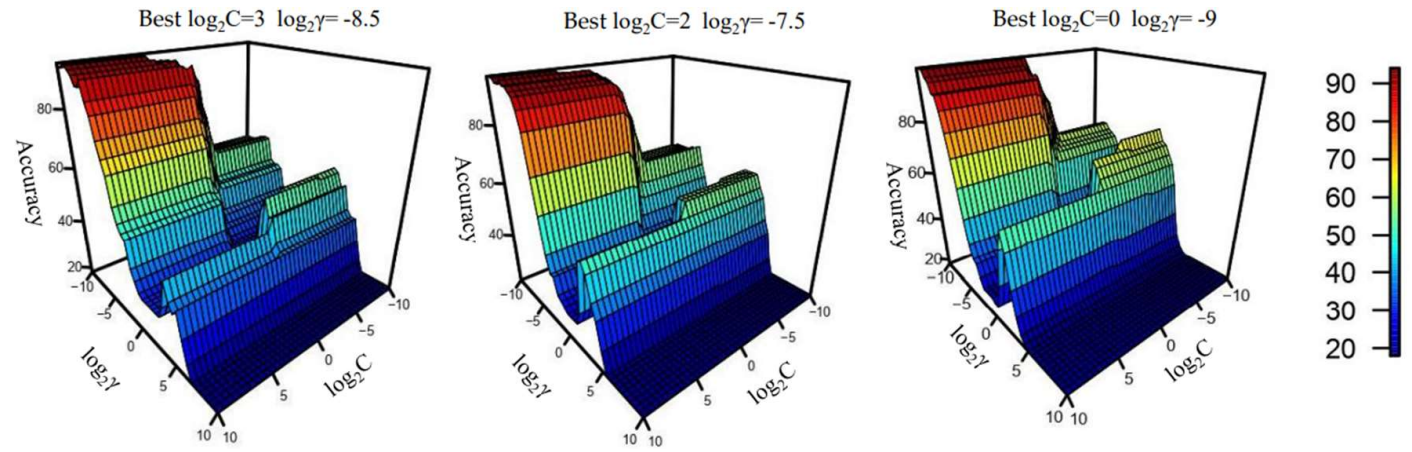

Figure 2. Search of best parameter for the SVM model using fivefold cross-validation: (a) stands for HS-IMS dataset; (b) stands for Vis-NIRS dataset; (c) stands for the joint HS-IMS/Vis-NIRS dataset.

A new model is made with the best hyperparameters selected however using cross validation leaving one out (LOOCV). Therefore, each sample of the dataset will be tested on the model. Table 1 shows the results of the accuracy rate on the dataset itself, for $\mathrm{LOOCV}$, as well as the name of the misclassified samples.

Table 1. Comparative classification results of the HS-IMS, the Vis-NIRS and the joint dataset results of the three datasets, based on SVM model.

\begin{tabular}{|c|c|c|c|}
\hline Dataset & $\begin{array}{c}\text { Accuracy rate } \\
\text { for test }(\%)\end{array}$ & $\begin{array}{l}\text { Accuracy rate for } \\
\text { LOOCV (\%) }\end{array}$ & $\begin{array}{c}\text { Misclassified samples } \\
\text { in LOOCV }\end{array}$ \\
\hline HS-IMS & 100 & 93.98 & $\begin{array}{c}\text { RS_5\%_2 } \\
\text { RS_100\%_1 } \\
\text { RS_100\%_2 } \\
\text { BS_50\%_1 } \\
\text { FS_25\%_1 }\end{array}$ \\
\hline Vis-NIRS & 100 & 93.98 & $\begin{array}{l}\text { IS_10\%_2 } \\
\text { IS_40\%_1 } \\
\text { RS_5\%_1 } \\
\text { BS_5\%_2 } \\
\text { FS_10\%_2 }\end{array}$ \\
\hline $\begin{array}{l}\text { Joint HS- } \\
\text { IMS/Vis-NIRS }\end{array}$ & 100 & 98.80 & FS_25\%_1 \\
\hline
\end{tabular}

As can be seen, the best result is obtained using the joint HS-IMS/Vis-NIRS dataset with only one sample misclassified in LOOCV. However, for each individual technique, a good classification result is also obtained. Therefore, it has been proved the suitability of the HS-IMS and Vis-NIRS combined with SVM for the detection of the adulteration in honeys.

\section{Conclusion}

This study attempts to detect whether a honey has been adulterated as well as the type of adulterant used, based on fast and low-cost techniques such as HS-IMS and Vis- 
NIRS, which could be a valid alternative to traditional analytical methods. The results obtained show a high classification rate for both systems, demonstrating the potential of these techniques when combined with SVM for honey quality control.

Author Contributions: Conceptualization, M.F.-G. and M.P.; methodology, M.J.A.-G., M.F.-G. and J.L.P.C.; software, J.L.P.C.; validation M.F.-G. and G.F.B.; formal analysis, M.J.A.-G. and J.L.P.C.; investigation, M.F.-G.; resources, M.F.-G. and G.F.B.; data curation, M.F.-G. and E.E.-B.; writingoriginal draft preparation, J.L.P.C. and M.J.A.-G.; writing-review and editing, M.F.-G., G.F.B. and M.P.; supervision, M.P.; funding acquisition M.F.-G. and G.F.B.; All authors have read and agreed to the published version of the proceeding.

Funding: This research received no external funding.

Acknowledgments: The authors would like to thank Granada Protected Designation of Origin (PDO) for providing the honey samples especially to Francisco José Orantes Bermejo (Director of Apinevada Laboratories).

Conflicts of Interest: The authors declare no conflict of interest.

\section{References}

1. Abu-Jdayil, B.; Al-Majeed Ghzawi, A.; Al-Malah, K.I.M.; Zaitoun, S. Heat effect on rheology of light- and dark-colored honey. J. Food Eng. 2002, 51, 33-38, doi:10.1016/S0260-8774(01)00034-6.

2. Official Journal of the European Communities, 2001. LL 10/47-L 10/52.12.1.2002. Council Directive 2001/110/EC of December 2001 Relating to Honey. Available online: Available online: https://eur-lex.europa.eu/legal-content/EN/ALL/?uri=CELEX\%3A32001L0110 (accessed on Dec 30, 2020).

3. Bontempo, L.; Camin, F.; Ziller, L.; Perini, M.; Nicolini, G.; Larcher, R. Isotopic and elemental composition of selected types of Italian honey. Meas. J. Int. Meas. Confed. 2017, 98, 283-289, doi:10.1016/j.measurement.2015.11.022.

4. Aliaño-González, M.J.; Ferreiro-González, M.; Espada-Bellido, E.; Palma, M.; Barbero, G.F. A screening method based on Visible-NIR spectroscopy for the identification and quantification of different adulterants in high-quality honey. Talanta 2019, 203, 235-241, doi:10.1016/j.talanta.2019.05.067.

5. Se, K.W.; Wahab, R.A.; Syed Yaacob, S.N.; Ghoshal, S.K. Detection techniques for adulterants in honey: Challenges and recent trends. J. Food Compos. Anal. 2019, 80, 16-32.

6. Aliaño-González, M.J.; Ferreiro-González, M.; Espada-Bellido, E.; Barbero, G.F.; Palma, M. Novel method based on ion mobility spectroscopy for the quantification of adulterants in honeys. Food Control 2020, 114, 107236, doi:10.1016/j.foodcont.2020.107236

7. Wang, S.; Guo, Q.; Wang, L.; Lin, L.; Shi, H.; Cao, H.; Cao, B. Detection of honey adulteration with starch syrup by high performance liquid chromatography. Food Chem. 2015, 172, 669-674, doi:10.1016/j.foodchem.2014.09.044.

8. Song, X.; She, S.; Xin, M.; Chen, L.; Li, Y.; Heyden, Y. Vander; Rogers, K.M.; Chen, L. Detection of adulteration in Chinese monofloral honey using $1 \mathrm{H}$ nuclear magnetic resonance and chemometrics. J. Food Compos. Anal. 2020, 86, 103390, doi:10.1016/j.jfca.2019.103390.

9. Ruiz-Matute, A.I.; Soria, A.C.; Martínez-Castro, I.; Sanz, M.L. A new methodology based on GC-MS to detect honey adulteration with commercial syrups. J. Agric. Food Chem. 2007, 55, 7264-7269, doi:10.1021/jf070559j.

10. Sobrino-Gregorio, L.; Vilanova, S.; Prohens, J.; Escriche, I. Detection of honey adulteration by conventional and real-time PCR. Food Control 2019, 95, 57-62, doi:10.1016/j.foodcont.2018.07.037.

11. Zhu, X.; Li, S.; Shan, Y.; Zhang, Z.; Li, G.; Su, D.; Liu, F. Detection of adulterants such as sweeteners materials in honey using near-infrared spectroscopy and chemometrics. J. Food Eng. 2010, 101, 92-97, doi:10.1016/j.jfoodeng.2010.06.014.

12. Arroyo-Manzanares, N.; García-Nicolás, M.; Castell, A.; Campillo, N.; Viñas, P.; López-García, I.; Hernández-Córdoba, M. Untargeted headspace gas chromatography - Ion mobility spectrometry analysis for detection of adulterated honey. Talanta 2019, 205, 120123, doi:10.1016/j.talanta.2019.120123.

13. Jia, W.; Liang, G.; Tian, H.; Sun, J.; Wan, C. Electronic Nose-Based Technique for Rapid Detection and Recognition of Moldy Apples. Sensors 2019, 19, 1526, doi:10.3390/s19071526.

14. Dankowska, A.; Kowalewski, W. Tea types classification with data fusion of UV-Vis, synchronous fluorescence and NIR spectroscopies and chemometric analysis. Spectrochim. Acta - Part A Mol. Biomol. Spectrosc. 2019, 211, 195-202, doi:10.1016/j.saa.2018.11.063. 